\title{
Calculation of the forbidden electric tensor polarizabilities of free Cs atoms and of Cs atoms trapped in a solid ${ }^{4} \mathrm{He}$ matrix
}

\author{
A. Hofer, ${ }^{*}$ P. Moroshkin, S. Ulzega, ${ }^{\dagger}$ and A. Weis \\ Département de Physique, Université de Fribourg, Chemin du Musée 3, 1700 Fribourg, Switzerland
}

(Received 6 June 2007; revised manuscript received 25 October 2007; published 4 January 2008)

\begin{abstract}
We give a detailed account on our semiempirical calculations of the forbidden electric tensor polarizability $\alpha_{2}^{(3)}$ of the ground state of free Cs atoms and of Cs atoms implanted in a solid ${ }^{4} \mathrm{He}$ matrix. The results are compared with measurements of $\alpha_{2}^{(3)}$ in the free atoms [Europhys. Lett. 76, 1074 (2006)] and in He-trapped atoms [Phys. Rev. A 75, 042505 (2007)]. Features with respect to calculations by other authors are the inclusion of off-diagonal hyperfine interactions and an analysis of contributions from continuum states, which turn out to be negligible. For both samples the results of the calculations are in good agreement with the experimental values, thereby settling a long-standing discrepancy.
\end{abstract}

PACS number(s): 31.15.xp, 32.10.Dk, 32.60.+i, 67.25.D-

\section{INTRODUCTION}

The interaction of an atom with an external static electric field (Stark effect) is one of the fundamental interactions in atomic physics. In atoms with degenerate orbital momentum states of opposite parity the change of level energies induced by the electric field is linear in the field strength $\mathbb{E}$, the hydrogen atom being the most prominent example. In most atoms, however, the Stark shift is quadratic in the field strength, and the electric-field-induced shift of a magnetic hyperfine (hf) sublevel $|\gamma\rangle=\left|n L_{J}, F, M\right\rangle$ is commonly parametrized in terms of the (electrostatic) polarizability $\alpha(\gamma)$ as

$$
\Delta E(\gamma)=-\frac{1}{2} \alpha(\gamma) \mathbb{E}^{2}
$$

In second order perturbation theory, the polarizability of states $n L_{J}$ with $J \geq 1$ can be written as the sum of an $F$ - and $M$-independent scalar polarizability $\alpha_{0}^{(2)}$ and an $F$ - and $M$-dependent tensor polarizability $\alpha_{2}^{(2)}$, while states with $J$ $<1$ have only a scalar polarizability. For spherically symmetric states, such as the $n S_{1 / 2}$ and $n P_{1 / 2}$ states in alkalimetal atoms, the Stark effect is thus a purely scalar effect, meaning that all hyperfine sublevels $\left|n L_{1 / 2}, F, M\right\rangle$ experience the same common shift. However, an $F$ and $M$ dependence of the Stark shifts in the $\left|n S_{1 / 2}\right\rangle$ alkali-metal ground states was already found experimentally in the late 1950's and early 1960's [1,2], and thus indicated the existence of a (forbidden) tensor polarizability of those states.

In 1967 Sandars [3] could show that this forbidden polarizability can be explained by expanding the perturbation treatment to third order, considering the Stark and the hyperfine interactions as simultaneous perturbations. The corresponding third order polarizability can also be expressed in terms of an $F$-dependent third order scalar polarizability $\alpha_{0}^{(3)}(F)$ and an $F$ - and $M$-dependent third order tensor polar-

\footnotetext{
*adrian.hofer@unifr.ch

${ }^{\dagger}$ Present address: EPFL, Lausanne, Switzerland.

\#www.unifr.ch/physics/frap/
}

izability $\alpha_{2}^{(3)}(F, M)$. The polarizability in Eq. (1) can thus be written as

$$
\begin{aligned}
\alpha(\gamma)= & \alpha_{0}^{(2)}\left(n L_{J}\right)+\alpha_{2}^{(2)}\left(n L_{J}, F, M\right)+\alpha_{0}^{(3)}\left(n L_{J}, F\right) \\
& +\alpha_{2}^{(3)}\left(n L_{J}, F, M\right),
\end{aligned}
$$

where the superscripts refer to the order of perturbation, while the subscripts refer to the rotational symmetry (scalar or second rank tensor) of the interaction.

Sandars' expressions were evaluated numerically in $[2,4]$ under simplifying assumptions, and yielded values of $\alpha_{2}^{(3)}(F, M)$ for five alkali-metal isotopes whose (absolute) calculated values were systematically larger than the corresponding experimental values [4-7]. Recently we have remeasured the third order tensor polarizability $\alpha_{2}^{(3)}(F, M)$ of the Cs ground state in an all-optical atomic beam experiment [7], yielding good agreement with the measurements from the 1960 's $[4,6]$. We have also measured $\alpha_{2}^{(3)}(F, M)$ of cesium atoms implanted in the cubic phase of a ${ }^{4} \mathrm{He}$ crystal, yielding a value whose modulus is $\approx 10 \%$ larger than in the free atom [8]. In parallel we have reanalyzed and extended the theoretical expressions of the tensor polarizabilities [5]. We have further extended the third order perturbation calculation [3] by including off-diagonal hyperfine interactions, not considered in the earlier calculations, as well as contributions from higher-lying bound and continuum states. This has yielded a theoretical value for $\alpha_{2}^{(3)}$ of $\operatorname{Cs}\left(6 S_{1 / 2}\right)$, which is in good agreement with all existing experimental results.

As discussed in [5] we have uncovered in an earlier calculation [3] an error concerning the relative signs of the polarizabilities of the two ground state hyperfine levels. Recently we have given an experimental verification of the sign of our calculation and discussed its implication for the dynamic Stark shift of primary frequency standards by the blackbody radiation field [8].

In this paper we present more details of that calculation, whose results were already outlined in $[5,8]$. In addition we present a calculation of $\alpha_{2}^{(3)}$ for cesium atoms in a cubic ${ }^{4} \mathrm{He}$ crystal by evaluating the matrix-induced alterations of the atomic energies and of the electric dipole and hyperfine ma- 
trix elements in the frame of the so-called standard bubble model [9]. Here too we obtain an excellent agreement with the experimental values.

\section{THEORY}

The Stark effect, i.e., the interaction of an alkali-metal atom with an external electric field $\mathbb{E}$ is described by the Hamiltonian $H_{\mathrm{St}}=-\vec{d} \cdot \overrightarrow{\mathbb{E}}$, where $\vec{d}$ is the electric dipole operator of the valence electron. Because of parity conservation, the Stark interaction vanishes in first order, and the effect of the electric field on the atomic level structure appears only in the next higher order(s), yielding shifts that are quadratic in the applied field strength.

\section{A. Second order perturbation theory}

The second order energy perturbation of the magnetic hyperfine sublevel $|\beta\rangle=\left|6 S_{1 / 2}, F, M\right\rangle$ of the ground state is given by

$$
\Delta E^{(2)}(\beta)=\sum_{\gamma} \frac{\left|\left\langle\beta\left|H_{\mathrm{St}}\right| \gamma\right\rangle\right|^{2}}{E_{\beta}-E_{\gamma}}
$$

where, according to the selection rule $\Delta L= \pm 1$ imposed by the Stark operator, the sum is to be taken over all excited $P$ states $|\gamma\rangle=\left|n P_{J}, f, m\right\rangle$, including continuum states, and where $E_{\gamma}$ are the unperturbed energies of those states. Following [10] one can define an effective second order Stark operator

$$
{ }_{2} H_{\text {eff }}=(\vec{d} \cdot \overrightarrow{\mathbb{E}}){ }_{2} \lambda(\vec{d} \cdot \overrightarrow{\mathbb{E}}),
$$

in which the scalar projection operator ${ }_{2} \lambda$ is defined as

$$
{ }_{2} \lambda=\sum_{\gamma} \frac{|\gamma\rangle\langle\gamma|}{E_{\beta}-E_{\gamma}} .
$$

With this definition of ${ }_{2} \mathrm{H}_{\text {eff }}$ the second order energy shift $\Delta E^{(2)}$ of the state $|\beta\rangle$ is given by the expectation value

$$
\Delta E^{(2)}(\beta)=\left\langle\left.\beta\right|_{2} H_{\text {eff }} \mid \beta\right\rangle,
$$

similar to the expression from first order perturbation theory. Note that the superscripts on the right of $\Delta E^{(n)}$ and $\alpha^{(n)}$ and the subscripts on the left of ${ }_{n} H_{\text {eff }}$ and ${ }_{n} \lambda$ refer to the order of perturbation.

The interaction Hamiltonian can be factorized [10] into an electric-field-dependent part and a part which depends only on atomic properties by defining the components of two rank- $K$ tensor operators as

$$
\begin{gathered}
\mathcal{E}_{Q}^{(K)}=[\mathbb{E} \otimes \mathbb{E}]_{Q}^{(K)}=\sum_{q, q^{\prime}}\left\langle 11 q q^{\prime} \mid K Q\right\rangle \mathbb{E}_{q}^{(1)} \mathbb{E}_{q^{\prime}}^{(1)}, \\
\mathcal{D}_{Q}^{(K)}=\left[d^{(1)} \otimes{ }_{2} \lambda \otimes d^{(1)}\right]_{Q}^{(K)}=\underset{q, q^{\prime}}{\sum_{1}\left\langle 11 q q^{\prime} \mid K Q\right\rangle d_{q 2}^{(1)} \lambda d_{q^{\prime}}^{(1)},}
\end{gathered}
$$

where the $\left\langle 11 q q^{\prime} \mid K Q\right\rangle$ are Clebsch-Gordan coefficients and where $d_{q}^{(1)}$ and $\mathbb{E}_{q}^{(1)}$ are the spherical components of the dipole operator and the electric field, respectively. The effec- tive Stark operator can then be written as the sum

$$
{ }_{2} H_{\mathrm{eff}}=\sum_{K=0}^{2}(-1)^{K}{ }_{2} H_{\mathrm{eff}}^{(K)}={ }_{2} H_{\mathrm{eff}}^{(0)}+{ }_{2} H_{\mathrm{eff}}^{(2)}
$$

of its multipole components

$$
{ }_{2} H_{\mathrm{eff}}^{(K)}=\sum_{Q}(-1)^{Q} \mathcal{E}_{Q}^{(K)} \mathcal{D}_{-Q}^{(K)}
$$

The vector contribution vanishes since $\mathcal{E}^{(1)} \propto[\mathbb{E} \otimes \mathbb{E}]^{(1)} \propto \vec{E}$ $\times \vec{E}$. The scalar term $\mathcal{E}^{(0)} \propto[\mathbb{E} \otimes \mathbb{E}]^{(0)} \propto \overrightarrow{\mathbb{E}} \cdot \overrightarrow{\mathbb{E}}=\mathbb{E}^{2}$ depends only on the magnitude of the field, while the rank-2 tensor term ${ }_{2} H_{\text {eff }}^{(2)}$ depends on its orientation, as can be seen, e.g., from its $Q=0$ component $\mathcal{E}_{0}^{(2)} \propto 3 \mathbb{E}_{z}^{2}-\mathbb{E}^{2}=\mathbb{E}^{2}\left(3 \cos ^{2} \theta-1\right)$. With this notation, the second order Stark effect $\Delta E^{(2)}(\beta)$ can be written as the expectation value

$$
\Delta E^{(2)}(\beta)=\left\langle\beta\left|{ }_{2} H_{\text {eff }}^{(0)}+{ }_{2} H_{\text {eff }}^{(2)}\right| \beta\right\rangle .
$$

The Wigner-Eckart theorem implies that the matrix element $\left\langle n L_{J}\left|{ }_{2} H_{\text {eff }}^{(K)}\right| n L_{J}\right\rangle$ vanishes unless $0 \leq K \leq 2 J$, so that the tensor part $(K=2)$ of the interaction vanishes,

$$
\left\langle\left.\beta\right|_{2} H_{\mathrm{eff}}^{(2)} \mid \beta\right\rangle \propto\left\langle 6 S_{1 / 2}\left\|_{2} H_{\mathrm{eff}}^{(2)}\right\| 6 S_{1 / 2}\right\rangle \equiv 0
$$

for the spherically symmetric $6 S_{1 / 2}$ state. As a consequence, the Stark effect in the alkali-metal ground state treated in second order perturbation theory is a purely scalar effect which is parametrized in terms of the $F$ - and $M$-independent second order scalar polarizability $\alpha_{0}^{(2)}$ as

$$
\Delta E^{(2)}(\beta)=-\frac{1}{2} \alpha_{0}^{(2)} \mathbb{E}^{2} .
$$

This energy perturbation results in an overall shift of the ground state sublevels. The experimental value of $\alpha_{0}^{(2)}\left(6 S_{1 / 2}\right)$ is $9.98(2) \times 10^{3} \mathrm{~Hz} /(\mathrm{kV} / \mathrm{cm})^{2}$ [11], and is theoretically well understood at a level of $10^{-3}[12,13]$. The $F$ and $M$-dependent shifts of the ground state sublevels discussed below are approximately 5 and 7 orders of magnitude smaller than this global scalar shift.

\section{B. Third order perturbation theory}

As already mentioned, Sandars has shown [3] that the forbidden electric-field-induced lifting of the Zeeman degeneracy can be explained by extending the perturbation theory to third order, including simultaneously the Stark interaction and the hyperfine interactions in terms of the perturbation operator $W=H_{\mathrm{St}}+H_{\mathrm{hf}}^{F c}+H_{\mathrm{hf}}^{d d}+H_{\mathrm{hf}}^{q}$, which consists of the Stark $\left(H_{\mathrm{St}}\right)$, the hyperfine Fermi contact $\left(H_{\mathrm{hf}}^{\mathrm{Fc}}\right)$, the hyperfine dipole-dipole $\left(H_{\mathrm{hf}}^{\mathrm{dd}}\right)$, and the hyperfine quadrupole $\left(H_{\mathrm{hf}}^{q}\right)$ operators. In [5] we have given explicit expressions of the hyperfine operators in terms of irreducible tensor operators.

Following the general rules of perturbation theory the third order energy perturbation of the ground state level $|\beta\rangle$ is given by 


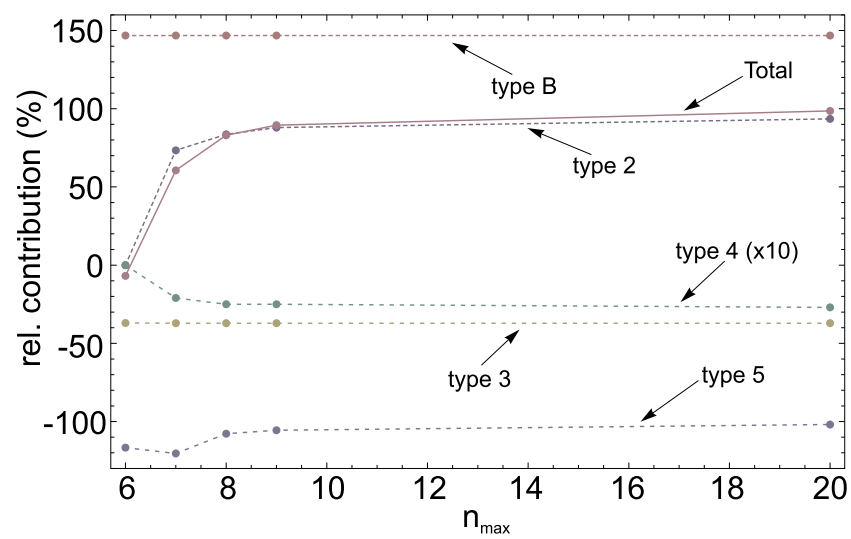

FIG. 1. (Color online) Dependence of the different contributions to the perturbation sum (14) leading to the tensor polarizability on $n_{\max }$, the maximum principal quantum number considered in the sum. Note that previous calculations took only diagonal (type $B$ ) hyperfine interactions into account.

$$
\begin{aligned}
\Delta E^{(3)}(\beta)= & \sum_{\gamma \neq \beta, \delta \neq \beta} \frac{\langle\beta|W| \gamma\rangle\langle\gamma|W| \delta\rangle\langle\delta|W| \beta\rangle}{\left(E_{\beta}-E_{\gamma}\right)\left(E_{\beta}-E_{\delta}\right)} \\
& -\langle\beta|W| \beta\rangle \sum_{\gamma \neq \beta} \frac{|\langle\gamma|W| \beta\rangle|^{2}}{\left(E_{\beta}-E_{\gamma}\right)^{2}},
\end{aligned}
$$

where $E_{\gamma}$ and $E_{\delta}$ are the unperturbed state energies.

The two terms of Eq. (14) are trilinear forms of the perturbation operator $W$, of which only terms proportional to $\mathbb{E}^{2}$ give nonzero contributions to the Stark interaction. As discussed by Ulzega et al. [5] the ground state hyperfine interaction $E_{\mathrm{hf}}(6 S)$ is factored out from the sum over pure Stark matrix elements in the second term of Eq. (14). This leads to $M$-independent, but $F$-dependent shifts of the ground state levels which can be parametrized, according to Eqs. (1) and (2), by a third order scalar polarizability $\alpha_{0}^{(3)}(F)$. The second term thus does not contribute to the tensor polarizability, but gives the leading contribution (type A interactions in the notation of [5]) to the Stark shift of the hyperfine (clock transition) frequency $[14,15]$. The contribution from this term is thus suppressed by a factor on the order of $E_{\mathrm{hf}}(6 S) /$ $\Delta E_{6 P-65} \approx 10^{-5}$ with respect to the second order scalar polarizability $\alpha_{0}^{(2)}$.

As discussed by Ulzega et al. [5] the first term of Eq. (14) has contributions from both diagonal and off-diagonal hyperfine matrix elements. The diagonal contributions (type $B$ interactions in the notation of [5]) dominate (see Fig. 1) and are suppressed by a factor on the order of $E_{\mathrm{hf}}(6 P) / \Delta E_{6 P-6 S}$ $\approx 10^{-7}$ with respect to the second order scalar shift $\alpha_{0}^{(2)}$. The Zeeman splitting of the ground state levels by the electric field is thus approximately 100 times smaller than the shift of the clock transition frequency.

The third order perturbation can again be expressed in terms of an effective Hamiltonian

$$
{ }_{3} H_{\mathrm{eff}}=(\vec{d} \cdot \overrightarrow{\mathbb{E}})_{3} \lambda(\vec{d} \cdot \overrightarrow{\mathbb{E}}),
$$

in which the projection operator ${ }_{3} \lambda$ is given by

$$
{ }_{3} \lambda=\sum_{\gamma} \frac{|\gamma\rangle\left\langle\gamma\left|H_{\mathrm{hf}}\right| \gamma\right\rangle\langle\gamma|}{\left(E_{\beta}-E_{\gamma}\right)^{2}} .
$$

The effective Hamiltonian can be expressed as the sum of a scalar and a rank-2 tensor, yielding the third order energy perturbation

$$
\Delta E^{(3)}(\beta)=\left\langle\beta\left|{ }_{2} H_{\mathrm{eff}}^{(0)}+{ }_{2} H_{\mathrm{eff}}^{(2)}\right| \beta\right\rangle,
$$

in a form equivalent to Eq. (11). The scalar part turns out to have the same $F$ dependence as the third order scalar polarizability $\alpha_{0}^{(3)}(F)$ from the second term of Eq. (14) and gives a correction to the latter on the order of $1 \%$.

By applying the Wigner-Eckart theorem, the contribution of the second rank tensor part can be written as

$$
\begin{gathered}
\left\langle\left.\beta\right|_{2} H_{\mathrm{eff}}^{(2)} \mid \beta\right\rangle \propto\left[3 M^{2}-F(F+1)\right]\left(3 \mathbb{E}_{z}^{2}-\mathbb{E}^{2}\right) \times\left\langle F \|\left[d^{(1)} \otimes{ }_{3} \lambda\right.\right. \\
\left.\left.\otimes d^{(1)}\right]^{(2)} \| F\right\rangle .
\end{gathered}
$$

The electronic and nuclear angular momenta in this equation cannot be decoupled since the operator $\left[d^{(1)} \otimes{ }_{3} \lambda \otimes d^{(1)}\right]^{(2)}$ depends explicitly on the hyperfine interaction term $\mathbf{J} \cdot \mathbf{I}$ and one can state in general that $\left\langle F\left\|\left[d^{(1)} \otimes{ }_{3} \lambda \otimes d^{(1)}\right]^{(2)}\right\| F\right\rangle \neq 0$ for states with $F \geq 1$. The tensor part has therefore an explicit $F$ and $M$ dependence, which can be parametrized in terms of a third order tensor polarizability $\alpha_{2}^{(3)}\left(6 S_{1 / 2}, F, M\right)$.

We can summarize the above results by parametrizing the two terms of the third order interaction [Eq. (14)] by a total third order polarizability as

$$
\Delta E^{(3)}=-\frac{1}{2} \alpha^{(3)}(F, M) \mathbb{E}^{2},
$$

with

$$
\alpha^{(3)}(F, M)=\alpha_{0}^{(3)}(F)+\alpha_{2}^{(3)}(F) \frac{3 M^{2}-F(F+1)}{2 I(2 I+1)} f(\theta) .
$$

The function $f(\theta)=3 \cos ^{2} \theta-1$ expresses the dependence of $\alpha^{(3)}$ on the angle $\theta$ between the electric field and the quantization axis.

\section{THIRD ORDER POLARIZABILITY OF THE FREE CESIUM ATOM}

\section{A. Earlier calculation revisited}

The leading term in the perturbation sum (14) is given by

$$
\begin{aligned}
\Delta E^{(3)}\left(6 S_{1 / 2}, F, M\right)= & \sum_{n, J, F, m}\left\langle n P_{J}, F\left|H_{\mathrm{hf}}\right| n P_{J}, F\right\rangle \\
& \times \frac{\left|\left\langle n P_{J}, F, m\left|H_{\mathrm{St}}\right| 6 S_{1 / 2}, F, M\right\rangle\right|^{2}}{\left(E_{n P_{J}, F, m}-E_{6 S_{1 / 2}, F, M}\right)^{2}},
\end{aligned}
$$

where we have used the fact that $H_{\mathrm{hf}}$ does not mix $F$ values, and where we have allowed for $M$ mixing by $H_{\mathrm{St}}$ in case the electric field is not along the quantization axis. This term is diagrammatically represented as diagram $B$ in [5], and we shall refer to it below as type $B$ interaction. The corresponding tensor polarizability of the $F=4$ state of Cs was evalu- 
ated in [4] considering - among other simplifying assumptions discussed in [5]—only diagonal hyperfine matrix elements in the $6 P_{1 / 2}$ and $6 P_{3 / 2}$ states. The result of that calculation is shown as point (f) in Fig. 4 and is in disagreement with the experimental results. We have redone this calculation after dropping all simplifying assumptions of [4], while still considering only diagonal matrix elements. We also used recent more precise experimental values [16] for the reduced dipole matrix elements $\left\langle 6 S_{1 / 2}\|d\| 6 P_{J}\right\rangle$, which yield the leading contribution. Point (g) in Fig. 4 represents the result of this reanalysis. As a result, the gap between theoretical and experimental values of the Cs tensor polarizability increases. Extending the perturbation sum to $n P_{J}$ states with $n>6$ does not affect the discrepancy at a significant level (line $B$ in Table 5 and Fig. 1).

\section{B. Inclusion of off-diagonal hf matrix elements}

All previous third order calculations have considered only diagonal hyperfine matrix elements (diagram $\mathbf{B}$ in Fig. 1 of [5]). However, the first term of the general third order expression [Eq. (14)] contains also off-diagonal hyperfine matrix elements. In [5] we have identified five different types of off-diagonal hyperfine mixing contributions:

(1) Type 1: $n$ mixing of $n S_{1 / 2}$ states.

(2) Type 2: $n$ mixing of $n P_{J}$ states with given $J$.

(3) Type 3: $J$ mixing of $n P_{J}$ states with given $n$.

(4) Type 4: $n$ and $J$ mixing of $n P_{J}$ states.

(5) Type 5: mixing of $m D_{3 / 2}$ and $6 S_{1 / 2}$ states.

Note that the type 1 interactions have an $F$, but no $M$ dependence and contribute thus only to the clock frequency shift. The different mixing types are represented as diagrams in Fig. 1 of [5] and the labeling used here follows the notation of that figure. The energy shifts due to interactions of types 2, 3, and 4 are given by sums of terms with the general structure

$$
\frac{\left\langle\beta\left|H_{\mathrm{St}}\right| \gamma_{1}\right\rangle\left\langle\gamma_{1}\left|H_{\mathrm{hf}}\right| \gamma_{2}\right\rangle\left\langle\gamma_{2}\left|H_{\mathrm{St}}\right| \beta\right\rangle}{\left(E_{\beta}-E_{\gamma_{1}}\right)\left(E_{\beta}-E_{\gamma_{2}}\right)},
$$

with $\quad|\beta\rangle=\left|n S_{1 / 2}, F, M\right\rangle, \quad\left|\gamma_{2}\right\rangle=\left|n P_{J}, f, m\right\rangle, \quad$ and $\quad\left|\gamma_{1}\right\rangle$ $=\left|n^{\prime} P_{J^{\prime}}, f, m\right\rangle$. The contributions to the interaction of type 5 have the form

$$
\frac{\left\langle\beta\left|H_{\mathrm{St}}\right| \delta\right\rangle\left\langle\delta\left|H_{\mathrm{St}}\right| \gamma\right\rangle\left\langle\gamma\left|H_{\mathrm{hf}}\right| \beta\right\rangle}{\left(E_{\beta}-E_{\delta}\right)\left(E_{\beta}-E_{\gamma}\right)},
$$

where $|\gamma\rangle=\left|m D_{3 / 2}, F, M\right\rangle$ and $|\delta\rangle=\left|n P_{J}, f, m\right\rangle$.

Some of the off-diagonal hyperfine matrix elements were considered earlier in calculations $[14,15,17]$ of the Stark shift of the $\left|6 S_{1 / 2}, F=3, M=0\right\rangle \rightarrow\left|6 S_{1 / 2}, F=4, M=0\right\rangle$ clock transition, but were never considered in a calculation of the tensor polarizability. The off-diagonal (type 1) matrix elements of the Fermi contact interaction between $S_{1 / 2}$ states contribute only to the clock frequency shift and are of no relevance here.

The (diagonal) matrix elements of the hyperfine quadrupole interaction $H_{\mathrm{hf}}^{q}$ are only relevant for states with $L, J$ $>1 / 2$ and their numerical values are two orders of magnitude smaller than all other hyperfine matrix elements. The matrix elements of the quadrupole part of the hyperfine Hamiltonian can be decomposed in a similar way. However, since their numerical evaluation yields only a relative contribution of $10^{-3}$ to the tensor polarizability we do not reproduce the corresponding algebraic expressions here. The only hyperfine operator contributing to the off-diagonal matrix elements is the dipole-dipole operator $H_{\mathrm{hf}}^{\mathrm{dd}}$, which can be expressed in terms of irreducible tensor operators as

$$
\mathrm{H}_{\mathrm{hf}}^{\mathrm{dd}}=a(r)\left(\mathbf{L}^{(1)}-\sqrt{10}\left[\mathbf{C}^{(2)} \otimes \mathbf{S}^{(1)}\right]^{(1)}\right) \cdot \mathbf{I}^{(1)},
$$

where $\mathbf{L}^{(1)}, \mathbf{S}^{(1)}$, and $\mathbf{I}^{(1)}$ are the irreducible vector operators associated with the orbital angular momentum, the electronic spin, and the nuclear spin, respectively, and $\mathbf{C}^{(k)}=\sqrt{\frac{4 \pi}{2 k+1}} \mathbf{Y}^{(k)}$ are the normalized spherical harmonic operators of rank $k$. The radial dependence of the hyperfine operator is given by $a(r)$. The two terms in Eq. (24) correspond to the dipolar magnetic interaction of the nuclear spin I with the orbital $\left(\propto \mathbf{L}^{(1)}\right)$ and the electronic spin $\left(\propto \mathbf{C}^{(2)} \times \mathbf{S}^{(1)}\right)$, respectively. The two interactions obey the selection rules $\Delta L=0$ and $\Delta L=0, \pm 2$, respectively. The relevant $\Delta L=0$ interactions (types 2,3,4) have nonvanishing off-diagonal matrix elements of the form $\left\langle\gamma_{1}\left|H_{\mathrm{hf}}^{\mathrm{dd}}\right| \gamma_{2}\right\rangle$ where $\left|\gamma_{i}\right\rangle=\left|n_{i} P_{J_{i}}, f, m\right\rangle$. The explicit reduction of those matrix elements leads to

$$
\begin{aligned}
\left\langle\gamma_{1}\left|H_{\mathrm{hf}}^{\mathrm{dd}}\right| \gamma_{2}\right\rangle= & (-1)^{f+L_{1}+J_{2}+1} 3 \sqrt{7} \sqrt{\left(2 J_{1}+1\right)\left(2 J_{2}+1\right)} \\
& \times\left\{\begin{array}{ccc}
J_{1} & 7 / 2 & f \\
7 / 2 & J_{2} & 1
\end{array}\right\}\left(\epsilon^{\text {orbital }}+\epsilon^{\text {sin }}\right)\langle a(r)\rangle,
\end{aligned}
$$

where we have separated the contribution of the orbital magnetic dipole interaction

$$
\epsilon^{\text {orbital }}=(-1)^{J_{2} 2} \sqrt{3}\left\{\begin{array}{ccc}
L_{1} & J_{1} & 1 / 2 \\
J_{2} & L_{2} & 1
\end{array}\right\} \delta_{L_{1}, L_{2}},
$$

from that of the spin dipolar interaction

$$
\begin{aligned}
\epsilon^{\text {spin }}= & (-1)^{7 / 2} 3 \sqrt{10} \sqrt{\left(2 L_{1}+1\right)\left(2 L_{2}+1\right)}\left(\begin{array}{ccc}
L_{1} & 2 & L_{2} \\
0 & 0 & 0
\end{array}\right) \\
& \times\left\{\begin{array}{ccc}
L_{1} & L_{2} & 2 \\
1 / 2 & 1 / 2 & 1 \\
J_{1} & J_{2} & 1
\end{array}\right\} .
\end{aligned}
$$

The coupling constant is given by

$$
\begin{aligned}
\langle a(r)\rangle & =\int_{0}^{\infty} \Psi_{n_{1} P_{J_{1}}}^{*} a(r) \Psi_{n_{2} P_{J_{2}}} r^{2} d r \\
& =\frac{2 g_{I}}{h} \frac{\mu_{0}}{4 \pi} \mu_{b}^{2} \int_{0}^{\infty} \Psi_{n_{1} P_{J_{1}}}^{*} \frac{1}{r^{3}} \Psi_{n_{2} P_{J_{2}}} r^{2} d r .
\end{aligned}
$$

Because of the second rank tensor character of the spherical harmonic operator $\mathbf{C}^{(2)}$ in Eq. (24), the spin dipolar term can also couple states with $\Delta L= \pm 2$ and can thus contribute to the third order Stark effect with off-diagonal matrix elements of the form $\left\langle\gamma\left|H_{\mathrm{hf}}^{\mathrm{dd}}\right| \beta\right\rangle$, given by Eq. (23), where $|\gamma\rangle$ $=\left|m D_{3 / 2}, F, M\right\rangle$ with $m \geq 5$ and $|\beta\rangle=\left|6 S_{1 / 2}, F, M\right\rangle$. In the latter case $\epsilon^{\text {orbital }}$ vanishes and Eq. (25) reduces to 


$$
\left\langle\gamma\left|H_{\mathrm{hf}}^{\mathrm{dd}}\right| \beta\right\rangle=\frac{\sqrt{5-F} \sqrt{F-2} \sqrt{F+3} \sqrt{F+6}}{4}\langle a(r)\rangle,
$$

where the corresponding coupling constant is given by

$$
\langle a(r)\rangle=\frac{2 g_{I}}{h} \frac{\mu_{0}}{4 \pi} \mu_{b}^{2} \int_{0}^{\infty} \Psi_{m D_{3 / 2}}^{*} \frac{1}{r^{3}} \Psi_{6 S_{1 / 2}} r^{2} d r
$$

\section{Electric dipole matrix elements}

Besides the hyperfine matrix elements the expressions involve matrix elements of the Stark interaction $H_{\mathrm{St}}$, i.e., of the electric dipole operator $d$ between $S$ and $P$ states. The latter can be reduced by applying the Wigner-Eckart theorem and the standard angular momentum decoupling rules, leading to the reduced matrix elements

$$
\begin{aligned}
\left\langle n S_{1 / 2}\|d\| m P_{J}\right\rangle & =(-1)^{J-(1 / 2)}\left\langle m P_{J}\|d\| n S_{1 / 2}\right\rangle \\
& =-\sqrt{\frac{2 J+1}{3}} D_{n S, m P_{J}},
\end{aligned}
$$

in which the radial integral is given by

$$
D_{n S, m P_{J}}=e \int_{0}^{\infty} R_{m P_{J}}(r) r^{3} R_{n S_{1 / 2}}(r) d r
$$

where $R_{n L_{J}}$ are the radial wave functions.

We note that the phases (signs) of the reduced matrix elements [Eq. (31)] are irrelevant for evaluating contributions involving diagonal hyperfine interactions, but are of fundamental importance for the contributions involving offdiagonal matrix elements.

Although dipole matrix elements between low-lying states in the Cs atoms can be calculated quite accurately using relativistic Hartree-Fock calculations (see, e.g., [19,21]) we have decided to rather use (more precise) experimental values, whenever they were available. Table I lists (in bold) the reduced dipole matrix elements used in the present calculation. For the matrix elements involving higher-lying states neither experimental nor theoretical values were available. In that case we have evaluated the corresponding radial integrals using nonrelativistic wave functions obtained by solving the Schrödinger equation as described in the next paragraph. The values of Table I show that this approach reproduces the matrix elements to better than $10 \%$. Assuming that the quoted accuracy also holds for excited states, and considering that the excited states give only a minor contribution to the final result (cf. Table I), we are confident that there is no significant uncertainty introduced by using our theoretical values for excited state matrix elements.

\section{Wave functions of the free cesium atom}

The nonrelativistic wave functions of the states $|n L, J\rangle$ can be separated into radial $R_{n L, J}(r)$ and angular $Y_{L, m}(\theta, \phi)$ parts

$$
\Psi_{n L_{J}}(\mathbf{r})=\langle\mathbf{r} \mid n L, J\rangle=R_{n L, J}(r) Y_{L, m}(\theta, \phi) .
$$

The radial wave functions are found as solutions of the radial Schrödinger equation for $u(r)=r R_{n, J}(r)$,
TABLE I. Radial integrals $D_{n S 1 / 2}, m P_{J}$ in atomic units. The values marked in bold were used in the calculation of the third order

\begin{tabular}{|c|c|c|c|}
\hline$n S_{1 / 2}, m P_{J}$ & Experiments & $\begin{array}{c}\text { Theory } \\
\text { (this work) }\end{array}$ & Deviation \\
\hline $6 S_{1 / 2}, 6 P_{1 / 2}$ & $\pm 5.5087(75)^{\mathrm{a}}$ & -5.3982 & $2 \%$ \\
\hline $6 S_{1 / 2}, 6 P_{3 / 2}$ & $\pm 5.4829(62)^{\mathrm{a}}$ & -5.3009 & $3 \%$ \\
\hline $6 S_{1 / 2}, 7 P_{1 / 2}$ & $\pm 0.3377(24)^{\mathrm{b}}$ & -0.3092 & $8 \%$ \\
\hline $6 S_{1 / 2}, 7 P_{3 / 2}$ & $\pm 0.5071(43)^{\mathrm{b}}$ & -0.4617 & $9 \%$ \\
\hline $7 S_{1 / 2}, 6 P_{1 / 2}$ & $\pm 5.184(27)^{\mathrm{C}}$ & +5.245 & $1 \%$ \\
\hline $7 S_{1 / 2}, 6 P_{3 / 2}$ & $\pm 5.611(27)^{\mathrm{c}}$ & +5.696 & $2 \%$ \\
\hline $7 S_{1 / 2}, 7 P_{1 / 2}$ & $\pm 12.625^{\mathrm{d}}$ & -13.298 & $5 \%$ \\
\hline $7 S_{1 / 2}, 7 P_{3 / 2}$ & $\pm 12.401^{\mathrm{d}}$ & -13.015 & $5 \%$ \\
\hline $6 S_{1 / 2}, 8 P_{1 / 2}$ & & -0.078 & \\
\hline $6 S_{1 / 2}, 8 P_{3 / 2}$ & & -0.130 & \\
\hline $6 S_{1 / 2}, 9 P_{1 / 2}$ & & -0.045 & \\
\hline $6 S_{1 / 2}, 9 P_{3 / 2}$ & & -0.078 & \\
\hline $6 S_{1 / 2}, 10 P_{1 / 2}$ & & $-\mathbf{0 . 0 3 0}$ & \\
\hline $6 S_{1 / 2}, 10 P_{3 / 2}$ & & -0.054 & \\
\hline
\end{tabular}
tensor polarizability. The theoretical values calculated from Schrödinger wave functions were used for matrix elements involving states with $n>7$. The theoretical values of the states involving $n=6,7$ were not used in the calculation but are shown merely to illustrate the accuracy of our calculation.

${ }^{a}$ Experimental values from Rafac et al. [16].

${ }^{\mathrm{b}}$ Experimental values from Vasilyev et al. [18].

${ }^{c}$ Experimental value quoted in [19].

${ }^{\mathrm{d}}$ Bennett et al. [20], with signs determined from Eq. (31).

$$
-\frac{1}{2} \frac{d^{2} u(r)}{d r}+\left[V_{\mathrm{CS}}^{\mathrm{free}}(r)+\frac{L(L+1)}{2 r^{2}}\right] u(r)=E u(r) .
$$

For the potential $V_{\mathrm{Cs}}^{\mathrm{free}}(r)$ we have used a scaled ThomasFermi model potential $V_{\mathrm{TF}}(r, \lambda)$ (with a scaling parameter $\lambda$ ) including dipolar and quadrupolar core-polarization corrections $V_{\text {pol }}(r)$ and the spin-orbit interaction $V_{\text {spin-orbit }}(r)$ that depends on the angular momentum $L$,

$$
V_{\mathrm{Cs}}^{\mathrm{free}}(r)=V_{\mathrm{TF}}(r, \lambda)+V_{\mathrm{pol}}(r)+V_{\text {spin-orbit }}(r) \text {. }
$$

This model follows the work of Gombas [22] and Norcross [23] and was explained in detail in [9]. Using this approach we have calculated the electronic wave functions for $n S_{J}$, $n P_{J}$, and $n D_{J}$ states up to $n=200$.

\section{E. Terms with diagonal hf matrix elements}

As stated above terms involving diagonal hyperfine matrix elements (hyperfine coupling constants) in the $n P_{J}$ states give the leading contribution to the third order tensor polarizability. In the numerical evaluation of the terms we use again experimental values, when they are available. In Table II we list the hyperfine coupling constants used (in bold). A comparison of experimental coupling constants of low-lying states with constants calculated using our Schrödinger wave functions is also made in order to illustrate the accuracy of the theoretical approach. As for the radial integrals there are 
TABLE II. Hyperfine coupling constants $A_{\mathrm{hf}}\left(n L_{J}\right)$ in MHz. The values marked in bold were used in the calculation of the third order tensor polarizability, where they contribute as diagonal matrix elements of $H_{\mathrm{hf}}$ to type $B$ interactions. The theoretical values calculated from Schrödinger wave functions were used for states with $n>8$. The theoretical values of the states $n=6, \ldots, 8$ were not used in the calculation but are shown to illustrate the accuracy of our calculation. The hyperfine constants $A_{n L_{J}}$ are related to the coupling constants $a(r)$ defined in the text by the relation $\langle a(r)\rangle=\frac{J(J+1)}{L(L+1)} A_{n L_{J}}$.

\begin{tabular}{lllc}
\hline \hline$n L_{J}$ & Experiments & Theory & Deviation \\
\hline $6 P_{1 / 2}$ & $\mathbf{2 9 1 . 9 2 0}(\mathbf{1 9})^{\mathrm{b}}$ & 317.9 & $8 \%$ \\
$6 P_{3 / 2}$ & $\mathbf{5 0 . 2 7 5}(\mathbf{3})^{\mathrm{c}}$ & 48.3 & $4 \%$ \\
$7 P_{1 / 2}$ & $\mathbf{9 4 . 3 5}(\mathbf{4})^{\mathrm{a}}$ & 99.9 & $6 \%$ \\
$7 P_{3 / 2}$ & $\mathbf{1 6 . 6 0 5}(\mathbf{6})^{\mathrm{a}}$ & 15.4 & $7 \%$ \\
$8 P_{1 / 2}$ & $\mathbf{4 2 . 9 7}(\mathbf{1 0})^{\mathrm{a}}$ & 45.0 & $5 \%$ \\
$8 P_{3 / 2}$ & $\mathbf{7 . 5 8}(\mathbf{1})^{\mathrm{a}}$ & 7.0 & $7 \%$ \\
$9 P_{1 / 2}$ & & $\mathbf{2 4 . 2}$ & \\
$9 P_{3 / 2}$ & & $\mathbf{3 . 8}$ & \\
$10 P_{1 / 2}$ & & $\mathbf{1 4 . 5}$ & \\
$10 P_{3 / 2}$ & & $\mathbf{2 . 3}$ & \\
$11 P_{1 / 2}$ & & $\mathbf{9 . 4}$ & \\
$11 P_{3 / 2}$ & & $\mathbf{1 . 5}$ & \\
\hline \hline
\end{tabular}

${ }^{\mathrm{a}}$ Experimental value taken from Arimondo et al. [24].

${ }^{\mathrm{b}}$ Experimental value taken from Rafac et al. [25].

${ }^{\mathrm{c}}$ Experimental value taken from Tanner et al. [26].

no experimental data for the high-lying states and we used our theoretical values, and, again, our accuracy is sufficient since it affects only the (very small) contributions from excited states.

It can be easily seen that the off-diagonal contributions are sensitive to the sign of the radial matrix elements. Whenever we extracted such matrix elements from experimental data-which all determined squared matrix elements-we have used the sign of the dipole matrix elements given by Eq. (31).

\section{F. Terms with off-diagonal hf matrix elements}

$S$-mixing off-diagonal hyperfine matrix elements have played an important role in the measurement of parity viola-
TABLE IV. Off-diagonal hyperfine coupling constants $\langle a(r)\rangle$ (in $\mathrm{MHz}$ ) of type 5 interactions. The numerical evaluation is based on Schrödinger wave functions as described in the text.

\begin{tabular}{lc}
\hline \hline$n$ & $\left\langle m D_{3 / 2}|a(r)| 6 S_{1 / 2}\right\rangle$ \\
\hline 5 & 74.4 \\
6 & 42.9 \\
7 & 28.4 \\
8 & 21.2 \\
9 & 16.4 \\
\hline \hline
\end{tabular}

tion in the Cs atom and they were measured and calculated with a high accuracy. However, such matrix elements intervene only in type 1 interactions, and do thus not contribute to the present calculation, in which the relevant terms come from $n P_{J}-n^{\prime} P_{J^{\prime}}$ mixing (types 2, 3, and 4) and $6 S_{1 / 2}-m D_{3 / 2}$ mixing (type 5). We have evaluated all relevant diagonal and off-diagonal hyperfine matrix elements using Schrödinger wave functions and the expressions given above. A selection of numerical values of those matrix elements for the lowestlying states is shown in Tables III and IV.

\section{G. Contribution of continuum states}

In principle, the summation in Eq. (14) has to be carried out over continuum states as well as over bound states. It was shown in $[14,15]$ that the continuum states contribute significantly $(\sim 15 \%)$ to type 1 interactions, which themselves do not contribute to the tensor polarizability. In order to estimate the influence of the continuum on the tensor polarizability we calculated the (positive energy) continuum wave functions using our Schrödinger approach and used them to evaluate bound-continuum and continuumcontinuum matrix elements of the electric dipole and hyperfine interactions.

For the numerical evaluation of transition matrix elements $\left\langle p, L_{J^{\prime}}^{\prime}|W| n L_{J}\right\rangle$ between bound $\left|n, L_{J}\right\rangle$ and continuum states $\left|p, L_{J^{\prime}}^{\prime}\right\rangle$ as well as between continuum states we use the expressions (32) and (28). We extended the perturbation sums (integrals) of all relevant interactions (types $B, 2-5$ ) over bound and continuum states.

TABLE III. Off-diagonal hyperfine coupling constants $\langle a(r)\rangle$ (in $\mathrm{MHz}$ ) representing interactions of types 2, 3, and 4. The numerical evaluation is based on Schrödinger wave functions as described in the text.

\begin{tabular}{lllllllll}
\hline \hline & $6 P_{1 / 2}$ & $7 P_{1 / 2}$ & $8 P_{1 / 2}$ & $9 P_{1 / 2}$ & $6 P_{3 / 2}$ & $7 P_{3 / 2}$ & $8 P_{3 / 2}$ & $9 P_{3 / 2}$ \\
\hline $6 P_{1 / 2}$ & & 785.3 & 827.1 & 386.4 & 187.7 & 106.1 & 71.5 & 52.5 \\
$7 P_{1 / 2}$ & & & 422.2 & 309.4 & 105.2 & 85.0 & 57.3 & 42.1 \\
$8 P_{1 / 2}$ & & & & 290.7 & 70.6 & 57.1 & 53.8 & 39.5 \\
$9 P_{1 / 2}$ & & & & & 51.8 & 41.8 & 39.4 & 41.3 \\
$6 P_{3 / 2}$ & 187.7 & 105.2 & 70.6 & 51.8 & & 14.3 & 9.6 & 7.1 \\
$7 P_{3 / 2}$ & 106.1 & 85.0 & 57.1 & 41.8 & & & 7.8 & 5.7 \\
$8 P_{3 / 2}$ & 71.5 & 57.3 & 53.8 & 39.4 & & & & 5.4 \\
$9 P_{3 / 2}$ & 52.5 & 42.1 & 39.5 & 41.3 & & & & \\
\hline \hline
\end{tabular}


TABLE V. Relative contributions (in $\%$ ) to $\alpha_{2}^{(3)}(F=4)$ from diagonal, type $B$, Eq. (21) and off-diagonal, types 2-5, Eqs. (22) and (23), hyperfine interactions of excited states $n L_{J}$.

\begin{tabular}{lllllll}
\hline \hline $\begin{array}{l}n \\
\text { Type }\end{array}$ & 6 & 7 & 8 & 9 & $10-200$ & Total \\
\hline$B$ & 146.8 & $\approx 0$ & $\approx 0$ & $\approx 0$ & $\approx 0$ & 146.8 \\
2 & & 73.4 & 10.2 & 4.3 & 6.4 & 94.3 \\
3 & -37.0 & -0.1 & $\approx 0$ & $\approx 0$ & $\approx 0$ & -37.1 \\
4 & & -2.1 & -0.4 & $\approx 0$ & -0.2 & -2.7 \\
5 & -116.7 & -3.7 & 12.6 & 2.2 & 4.3 & -101.3 \\
Total & -6.9 & 67.5 & 22.4 & 6.5 & 10.5 & 100.0 \\
\hline \hline
\end{tabular}

The calculation poses no convergence problems, except for the type 5 interaction, which is the only one that involves dipole matrix elements between continuum states. Such matrix elements also occur, e.g., in the calculation of bremsstrahlung transitions, and pose some technical difficulty. For their evaluation we have used the method of exterior complex scaling introduced in $[27,28]$ with a numerical implementation on a grid. The regular and outgoing waves in the complex plane were obtained through a step-by-step propagation using recursive relations [27]. As a result we find a total contribution from continuum states to the final value of $\alpha_{2}^{(3)}$ on the order of $0.2 \%$.

\section{H. Numerical evaluation of the third order tensor polarizability for the free Cs atom}

We have done a numerical evaluation of all diagonal (type $B$ ) and off-diagonal (types 2-5) contributions to the tensor polarizability summing over bound states up to $n=200$. For the $F=4$ state we find

$$
\alpha_{2}^{(3)}(F=4)=-3.72(25) \times 10^{-2} \mathrm{~Hz} /(\mathrm{kV} / \mathrm{cm})^{2},
$$

in which the contribution from the quadrupole hyperfine interaction is $2 \times 10^{-5} \mathrm{~Hz} /(\mathrm{kV} / \mathrm{cm})^{2}$. This result is shown as a dotted line in the left part of Fig. 4, together with previous theoretical and experimental results, described in the figure caption.

Table $\mathrm{V}$ shows the relative contributions of the different diagonal (type $B$ ) and off-diagonal (types 2-5) hyperfine interactions to the third order tensor polarizability. One notes that the diagonal (type $B$ ) contributions which were the only ones used in previous calculations overestimate the modulus of the third order tensor polarizability by approximately $50 \%$. Of all the off-diagonal contributions the interactions of type 2 and 5 (n-mixing of $n P_{J}$ states with given $J$ and $6 S_{1 / 2}-n D_{3 / 2}$ mixing) give large contributions with opposite signs which cancel each other to a large extent.

We estimate the uncertainty of the final result to be $\approx 7 \%$. This estimation is based on the precision (2-9\%) with which our wave functions reproduce measured hyperfine constants (Table II) and dipole matrix elements (Table I), and considers that we have used (more precise) experimental values for the leading terms. As shown in the previous paragraph the relative contribution of the continuum states to the tensor polarizability is on the order of $10^{-3}$ and is thus neg- ligible in the final result. Figure 1 shows the relative contributions from the different interactions in a cumulative way.

Our theoretical value for $\alpha_{2}^{(3)}(F=4)$ of the free Cs atom is in good agreement with all experimental values (Fig. 4).

\section{THIRD ORDER POLARIZABILITY OF CESIUM IN SOLID HELIUM}

\section{A. Experiment}

In connection with a proposed search for an electric dipole moment of $\mathrm{Cs}$ atoms in solid helium we were led to study the tensor Stark shifts in that unusual sample. The exceptionally long longitudinal and transverse spin relaxation times of $\mathrm{Cs}$ in the body-centered cubic (bcc) phase of ${ }^{4} \mathrm{He}$ crystals form the basis for high resolution magnetic resonance experiments. Recently we have measured the Stark shift of magnetic resonance lines in the ground state of $\mathrm{Cs}$ atoms implanted in bcc ${ }^{4} \mathrm{He}$ by two different techniques [8]. The experimental values of the corresponding tensor polarizabilities $-4.07(20) \times 10^{-2} \mathrm{~Hz} /(\mathrm{kV} / \mathrm{cm})^{2}$ and $-4.36(28) \times 10^{-2} \mathrm{~Hz} /(\mathrm{kV} / \mathrm{cm})^{2}$ for $\alpha_{2}^{(3)}(F=4)$ are shown as points $(d)$ and $(e)$ in Fig. 4.

\section{B. Wave function of $\mathrm{Cs}$ in solid ${ }^{4} \mathrm{He}$}

For calculating the tensor polarizability of Cs in solid helium one has to include the influence of the He matrix on the atomic energies and wave functions. Because of the Pauli repulsion $\mathrm{Cs}$ atoms form spherical bubbles, which are described by a spherically symmetric continuous He density distribution

$$
\rho\left(R, R_{0}, \epsilon\right)= \begin{cases}0, & R<R_{0} \\ \rho_{0}\left\{1-\left[1+\epsilon\left(R-R_{0}\right)\right] e^{-\epsilon\left(R-R_{0}\right)}\right\} & R, \geq R_{0},\end{cases}
$$

where $R_{0}$ is the bubble radius. $\epsilon$ describes the steepness of the interface (density changes from zero to the bulk density) and $\rho_{0}$ is the bulk density which depends on the He temperature and pressure. In Eq. (37) we have assumed that solid helium is an incompressible fluid, an assumption well justified by the quantum nature of condensed ${ }^{4} \mathrm{He}$. Our calculation of the Cs wave functions relies on an extension of the so-called bubble model [9], which was shown in the past to be well suited for describing energies, absorption or emission 
line shapes, hyperfine structure, and lifetimes of alkali metals in solid helium [9,29].

The total interaction potential experienced by the Cs valence electron is given by

$$
\begin{aligned}
V_{\mathrm{Cs}}^{\mathrm{bub}}\left(r, R_{0}, \epsilon\right)= & V_{\mathrm{Cs}}^{\mathrm{free}}(r)+\int d^{3} R \rho\left(R, R_{0}, \epsilon\right) \\
& \times\left[V_{\mathrm{He}}(\mathbf{r}, \mathbf{R})+V_{\mathrm{cross}}(\mathbf{r}, \mathbf{R})+V_{\mathrm{cc}}(\mathbf{R})\right],
\end{aligned}
$$

where $V_{\mathrm{Cs}}^{\mathrm{free}}(\mathbf{r})$ is the potential of the valence electron with the $\mathrm{Cs}^{+}$core introduced in Eq. (35). The ionic core and the $\mathrm{He}$ atoms are assumed to have fixed spatial positions (BornOppenheimer approximation). In Eq. (38) $V_{\mathrm{He}}(\mathbf{r}, \mathbf{R})$ and $V_{\text {cc }}(\mathbf{R})$ represent the interactions of the valence electron and the $\mathrm{Cs}^{+}$ion with a He atom. The potential $V_{\text {cross }}(\mathbf{r}, \mathbf{R})$ describes the three-body interaction resulting in the simultaneous polarization of the $\mathrm{He}$ atom by the $\mathrm{Cs}$ core and the valence electron. $\mathbf{r}$ and $\mathbf{R}$ point from the core to the electron and to each He atom, respectively. Explicit forms for all the potentials as well as numerical parameter values are given in [9]. The potentials seen by the valence electron in the free Cs atom $V_{\mathrm{Cs}}^{\text {free }}$ and in the Cs atom trapped in solid $\mathrm{He} V_{\mathrm{Cs}}^{\text {bub }}$ are shown, together with the energies of the lowest states in Fig. 3.

The energy needed to from a bubble consists of a pressure volume term, a surface energy (with surface tension parameter $\sigma$ ), and the kinetic energy $E_{\text {kin }}$, which arises from the localization of the $\mathrm{He}$ atoms at the bubble interface

$$
E_{\mathrm{bub}}\left(R_{b}, \epsilon\right)=\frac{4}{3} \pi R_{b}^{3} p+4 \pi R_{b}^{2} \sigma+E_{\mathrm{kin}},
$$

where $R_{b}=f\left(R_{0}\right)$ is the center of gravity of the interface. The total energy of the bubble defect is thus $V_{\text {tot }}^{\mathrm{bub}}\left(r, R_{0}, \epsilon\right)$ $=V_{\mathrm{Cs}}^{\mathrm{bub}}\left(r, R_{0}, \epsilon\right)+E_{\mathrm{bub}}\left(R_{0}, \epsilon\right)$, and the average bubble radius $R_{b}$ is found by numerically minimizing the total energy with respect to the two parameters $R_{0}$ and $\epsilon$. Using the known bubble parameters and the interaction potential one can then solve the radial Schrödinger equation as for the valence electron of the free Cs atom. In order to illustrate the effect of the He bubble we compare in Fig. 2 the wave function of the $9 P_{1 / 2}$ state of the free Cs atom with the corresponding wave function in the bubble. As expected, the wave function in the bubble is compressed due to the repulsive interaction with the surrounding helium atoms.

Using the solutions (wave functions) one can then evaluate the matrix elements required for the numerical calculation of the third order polarizability, in analogy to the free atomic case.

\section{Numerical evaluation of the third order tensor polarizability of $\mathrm{Cs}$ in solid $\mathrm{He}$}

The numerical evaluation of the tensor polarizability $\alpha_{2}^{(3)}$ for $\mathrm{Cs}$ in solid $\mathrm{He}$ was done in analogy to the case of the free atom, with wave functions and energy levels calculated using the bubble model introduced in Sec. IV B. While in the case of the free Cs atom we have considered bound states up to

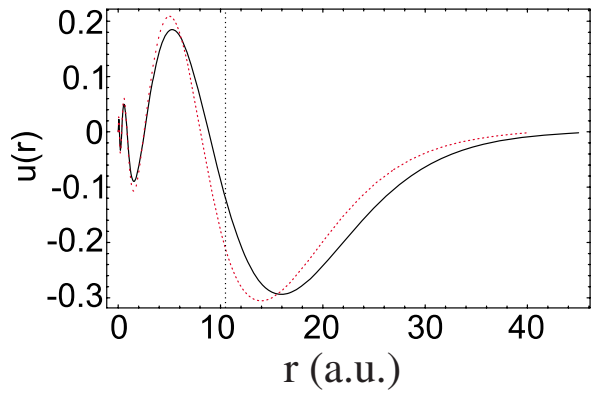

FIG. 2. (Color online) Comparison of the $9 P_{1 / 2}$ wave function of the free Cs atom (black solid line) with the same wave function of a $\mathrm{Cs}$ atom in a spherical helium bubble (red dotted line). The bubble parameters correspond to the equilibrium bubble shape of a ground state $\mathrm{Cs}$ atom $\left(R_{0}=10.2\right.$, indicated by the vertical dotted line and $\epsilon=2.45$ in atomic units).

the principal quantum number $n=200$ we need only to consider states up to $9 P$ in the He matrix, since higher-lying states are not bound (see Fig. 3). Moreover, the energy levels of the higher-lying states are strongly shifted to higher energies. For example, the energies of the $8 P_{1 / 2}$ and $9 P_{1 / 2}$ state are displaced by $5580 \mathrm{~cm}^{-1}(22 \%)$ and $5830 \mathrm{~cm}^{-1}(21 \%)$, respectively, with respect to the free atom. Since the perturbation sum involves squared energy denominators these excited states give smaller contributions than in the free atomic case. In Table VI we analyze the dependence of the relative contributions from the different diagrams on the number of states included in the perturbation sum, which qualitatively reflects the same features as in the free atomic case. Our final theoretical value of the tensor polarizability of Cs in solid ${ }^{4} \mathrm{He}$ is

$$
\alpha_{2}^{(3)}(F=4)=-4.11 \times 10^{-2} \mathrm{~Hz} /(\mathrm{kV} / \mathrm{cm})^{2}
$$

On the right of Fig. 4 we compare this theoretical value with the experimental values of $\alpha_{2}^{(3)}$ of Cs atoms trapped in a body-centered cubic solid ${ }^{4} \mathrm{He}$ matrix [8] and find an excellent agreement.

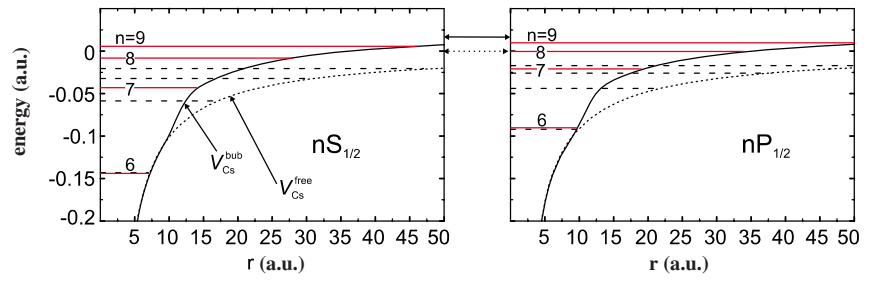

FIG. 3. (Color online) Energies of the lowest $n S_{1 / 2}$ (left) and $n P_{1 / 2}$ (right) states of the free Cs atom (dashed lines) and of Cs in the bcc solid ${ }^{4} \mathrm{He}$ (solid lines, $R_{0}=10.2, \epsilon=2.45$ atomic units). The corresponding potentials for the valence electron of the free $\mathrm{Cs}$ atom [Eq. (35)] and of the Cs atom in solid He [Eq. (38)] are shown as dotted and solid lines, respectively. The $n=9$ states are the highest bound states in the bubble. The bump in the potential $V_{\mathrm{Cs}}^{\mathrm{bub}}$ is due to the repulsion by the bubble interface. The arrows between the graphs indicate the ionization limits. 
TABLE VI. Relative contributions (in \%) to the tensor polarizability for $\mathrm{Cs}$ trapped in solid He. Note that $\mathrm{Cs}$ in solid He has no bound states with $n>9$.

\begin{tabular}{lllll}
\hline \hline & $n=6,7$ & $n=8$ & $n=9$ & Total \\
\hline$B$ & 143.4 & $\approx 0$ & $\approx 0$ & 143.4 \\
2 & 21.1 & 13.4 & 8.5 & 43.0 \\
3 & -26.8 & $\approx 0$ & $\approx 0$ & -26.8 \\
4 & -0.2 & -0.1 & -0.1 & -0.4 \\
5 & -65.4 & 4.1 & 2.1 & -59.2 \\
Total & 72.1 & 17.4 & 10.5 & 100.0 \\
\hline \hline
\end{tabular}

\section{SUMMARY}

We have presented calculational details of our semiempirical evaluation of the cesium ground state tensor polarizability $\alpha_{2}^{(3)}$. This $F$ - and $M$-dependent polarizability is forbidden in second order perturbation theory and arises only when considering the Stark effect and hyperfine interactions in a third order perturbation calculation. The tensor polarizability is suppressed by seven orders of magnitude with respect to the usual scalar polarizability of the atom.

We have evaluated $\alpha_{2}^{(3)}$ both for the free cesium atom and for $\mathrm{Cs}$ embedded in a cubic solid ${ }^{4} \mathrm{He}$ matrix using solutions of the Schrödinger equation with appropriate potentials for evaluating the relevant matrix elements in both cases. We have found that off-diagonal hyperfine matrix elements, which were not considered in previous treatments give substantial contributions (of different signs) to $\alpha_{2}^{(3)}$. As a result we obtain theoretical values for the tensor polarizabilities that are in excellent agreement with previous and recent

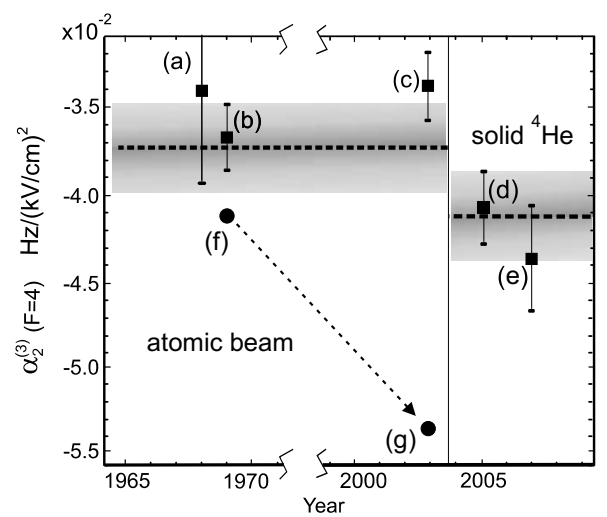

FIG. 4. The Cs tensor polarizability $\alpha_{2}^{(3)}(F=4)$. Atomic beam measurements of (a) Carrico et al. [6], (b) Gould et al. [4], and (c) Ospelkaus et al. [7]. Points (d) and (e) represent recent measurements in solid helium [8]. The circles are the theoretical values (f) from Gould et al. [4] and (g) from Ulzega et al. [5]. The dashed lines represent the results of our new calculations for the free atom and for Cs in a solid helium matrix, together with their uncertainties (shaded bands).

measurements. The modulus of the experimental tensor polarizability of $\mathrm{Cs}$ in the $\mathrm{He}$ matrix is found to be $8-10 \%$ larger than the one of the free Cs atom.

It thus seems that the 40 year old discrepancy between experimental and theoretical tensor polarizabilities has now found a satisfying final solution.

\section{ACKNOWLEDGMENT}

This work was supported by the Grant No. 200020103864 of the Schweizerischer Nationalfonds.
[1] R. D. Haun and J. R. Zacharias, Phys. Rev. 107, 107 (1957).

[2] E. Lipworth and P. G. H. Sandars, Phys. Rev. Lett. 13, 716 (1964).

[3] P. G. H. Sandars, Proc. Phys. Soc. London 92, 857 (1967).

[4] H. Gould, E. Lipworth, and M. C. Weisskopf, Phys. Rev. 188, 24 (1969).

[5] S. Ulzega, A. Hofer, P. Moroshkin, and A. Weis, Europhys. Lett. 76, 1074 (2006).

[6] J. P. Carrico, A. Adler, M. R. Baker, S. Legowski, E. Lipworth, P. G. H. Sandars, T. S. Stein, and C. Weisskopf, Phys. Rev. 170, 64 (1968).

[7] C. Ospelkaus, U. Rasbach, and A. Weis, Phys. Rev. A 67, 011402(R) (2003).

[8] S. Ulzega, A. Hofer, P. Moroshkin, R. Müller-Siebert, D. Nettels, and A. Weis, Phys. Rev. A 75, 042505 (2007).

[9] A. Hofer, P. Moroshkin, S. Ulzega, D. Nettels, R. MüllerSiebert, and A. Weis, Phys. Rev. A 76, 022502 (2007).

[10] J. R. P. Angel and P. G. H. Sandars, Proc. R. Soc. London, Ser. A 305, 125 (1968).

[11] J. M. Amini and H. Gould, Phys. Rev. Lett. 91, 153001 (2003).

[12] A. Derevianko and S. G. Porsev, Phys. Rev. A 65, 053403
(2002).

[13] H. L. Zhou and D. W. Norcross, Phys. Rev. A 40, 5048 (1989).

[14] K. Beloy, U. I. Safronova, and A. Derevianko, Phys. Rev. Lett. 97, 040801 (2006).

[15] E. J. Angstmann, V. A. Dzuba, and V. V. Flambaum, Phys. Rev. Lett. 97, 040802 (2006).

[16] R. J. Rafac, C. E. Tanner, A. E. Livingston, K. W. Kukla, H. G. Berry, and C. A. Kurtz, Phys. Rev. A 50, R1976 (1994).

[17] J. D. Feichtner, M. E. Hoover, and M. Mizushima, Phys. Rev. 137, A702 (1965).

[18] A. A. Vasilyev, I. M. Savukov, M. S. Safronova, and H. G. Berry, Phys. Rev. A 66, 020101(R) (2002).

[19] M. S. Safronova, W. R. Johnson, and A. Derevianko, Phys. Rev. A 60, 4476 (1999).

[20] S. C. Bennett, J. L. Roberts, and C. E. Wieman, Phys. Rev. A 59, R16 (1999).

[21] V. A. Dzuba, V. V. Flambaum, A. Y. Kraftmakher, and O. P. Sushkov, Phys. Lett. A 142, 373 (1989).

[22] P. Gombas, Pseudopotentiale (Springer-Verlag, BerlinGottingen-Heidelberg, 1956).

[23] D. W. Norcross, Phys. Rev. A 7, 606 (1973). 
[24] E. Arimondo, M. Inguscio, and P. Violino, Rev. Mod. Phys. 49, 31 (1977).

[25] R. J. Rafac and C. E. Tanner, Phys. Rev. A 56, 1027 (1997).

[26] C. E. Tanner and C. Wieman, Phys. Rev. A 38, 1616 (1988).
[27] M. Chrysos and M. Fumeron, J. Phys. B 32, 3117 (1999).

[28] M. Chrysos, J. Phys. B 33, 2875 (2000).

[29] P. Moroshkin, A. Hofer, S. Ulzega, and A. Weis, Fiz. Nizk. Temp. 32, 1297 (2006) [Low Temp. Phys. 32, 981 (2006)]. 